\title{
AN ANALYSIS OF FRETTING CRACKS-II. UNLOADING AND RELOADING PHASES
}

\author{
Sheri D. Sheppard, D. A. Hills $\dagger$ and J. R. Barber \\ Department of Mechanical Engineering and Applied Mechanics, University of Michigan, \\ Ann Arbor, MI 48109, U.S.A.
}

(Received 19 July 1984)

\begin{abstract}
An elastic half-plane containing a surface-breaking crack normal to the free surface. subjected to loading by uniform tractions over a given length of its surface, is considered. The tractions consist of pressure, constant in time, and a shear load, varying sinusoidally in time, bolh applied adjacent to the crack. This geometry approximates the classical fretting problem with a resulting fatigue crack. The faces of the crack are allowed to transmit Coulomb friction.

In this paper it is assumed that the pressure has already been applied, and that the shear traction has been increased continuously from zero to a maximum value. The effect of varying the shear traction through the rest of one load cycle is considered. Stress intensity factors are computed for various crack lengths, friction coefficients, and ratios of applied tractions. The history of stick and slip zones found along the crack faces is monitored.
\end{abstract}

\section{INTRODUCTION}

The geometry of the surface-breaking crack is shown in Fig. 1(a). The half-space $x>0$ is assumed to be loaded by constant normal pressure $p_{0}$ over a small portion $(0<y<$ $L)$ of its surface. A shear traction $q=\lambda p_{0}$ varying harmonically with time is then applied over $L$. This configuration is used to model a fatigue crack emanating from a fretting contact. References included in Part 1 [1] describe experiments which commonly result in this type of failure. It was expected that a severe stress intensity would develop at the crack tip as the shear traction was increased in the positive sense [Fig. 1(b)]. This was studied in Part 1. Although the absolute maximum stress intensity factor is certainly important and probably indicates the portion of the loading cycle where most crack growth occurs, it is the range of stress intensity which is required by a growth rate/stress intensity law such as that due to Forman[2]. Therefore, in the present paper we aim to extend the results of [1] by following the stress intensity experienced by the crack tip throughout its loading cycle. The four quadrants of one cycle of loading are shown in Fig. 1(b) and are denoted by Roman numerals. The vertical crack $0<$ $x<c$ ) is located along the $y=0$ axis, and during part of the cycle it may be open in the interval $0<x<a$.

It will be assumed in the present paper that the magnitude of $\lambda_{\max }$, the ratio of shear to normal tractive loads, is sufficiently great to open the crack to its tip in region I. It is felt that for practical purposes this is not likely to be a restriction, since a significant crack growth increment will be experienced when the crack is fully open and suffering combined modes $I$ and II loading, and this condition will therefore probably obtain for all cracks which are not experiencing self-arrest. A consequence of this assumption is that all residual shear tractions developed at the end of each cycle of loading will be relaxed out, and therefore the crack will experience the same interfacial tractions in the first loading cycle as in the steady state. To assist in our description of crack response throughout a cycle, it is convenient if we classify cracks as one of two types, as shown in Fig. 2. Thus, long cracks with a low coefficient of interfacial friction $f$, which fall below the dividing line shown, are denoted type $A$, while short cracks with high coefficients of friction will lie above that line and are hence denoted type $B$. Normalized crack lengths $(c / L)$ are used. In the following development, reference should be made to Table 1 .

† Permanent address: Department of Engineering Science, Oxford University, Parks Road, Oxford OXI 3PJ, U.K. 
(a).

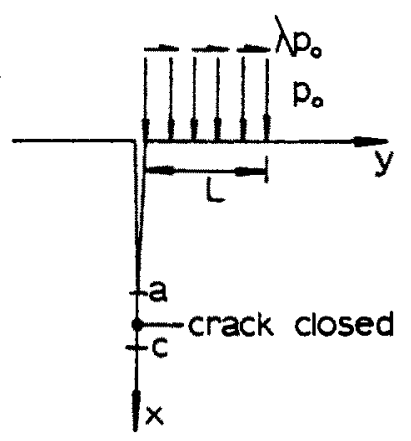

(b).

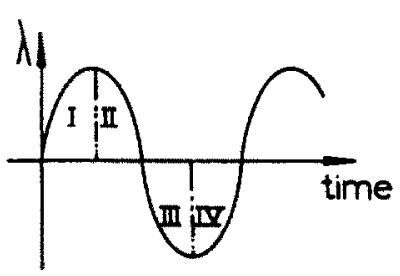

Fig. 1. Crack geometry and variation of $\lambda$ with time.

\section{REGION I}

We first summarize the crack behavior in region I, details of which are given in [1]. Initially, under the application of normal pressure alone, the crack slips along its entire length. Type $B$ cracks, when loaded by an increasing shear traction $\lambda p_{0}$, experience continued forward slip along their entire length and, consequently, an increasingly positive mode II stress intensity factor $\left(K_{11}\right)$. Eventually, the gap extends to the crack tip [i.e. $a$ coincides with $c$ in Fig. 1(a)], whereupon an opening mode stress

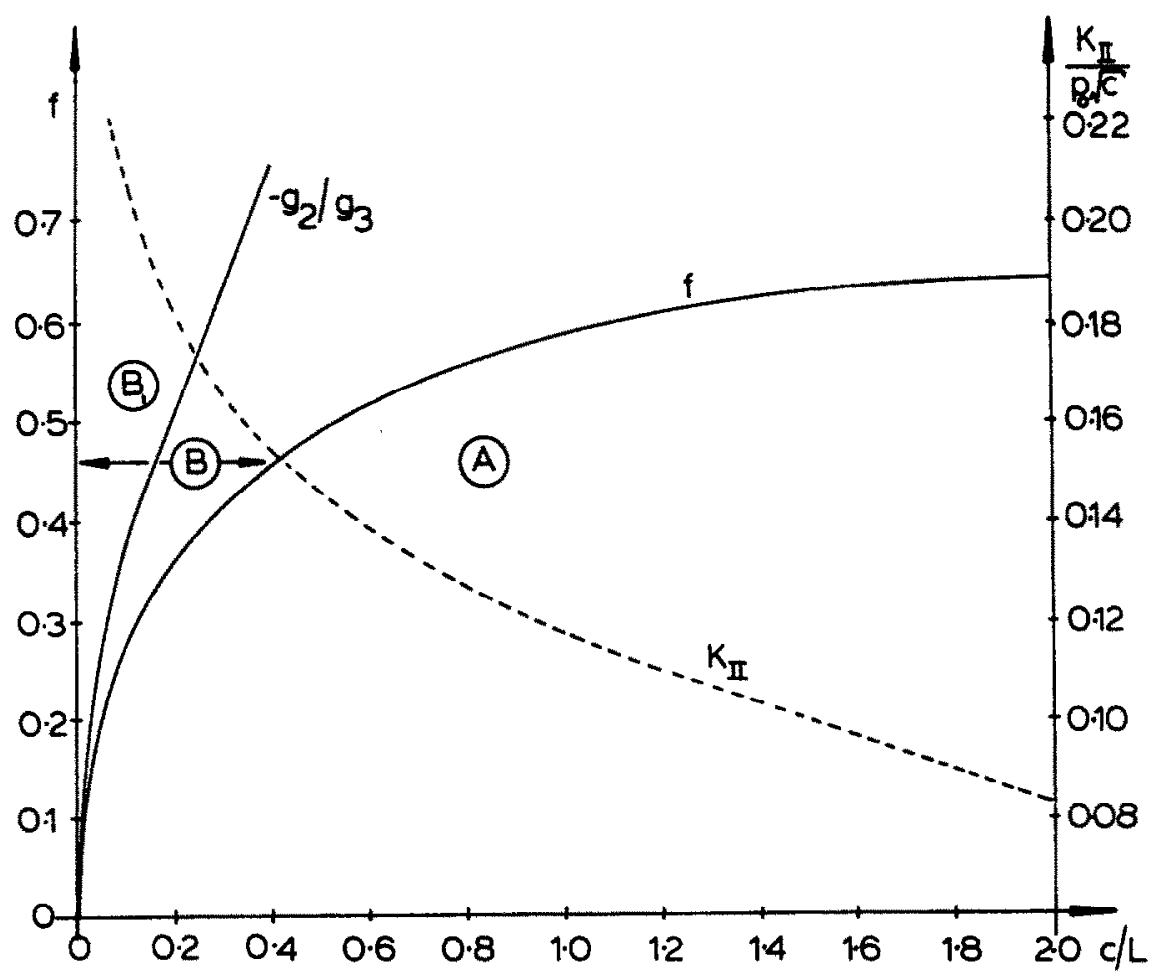

Fig. 2. Curve separating two types of crack response found. Also shown is $K_{11}$ for type $A$ cracks which are just on the point of sticking during region 11 . 
Table 1. Loading history and crack response

\begin{tabular}{|c|c|c|c|c|c|c|c|c|c|}
\hline \multicolumn{2}{|c|}{ Crack } & \multicolumn{2}{|c|}{ Pressure alone } & \multicolumn{2}{|l|}{ Region I } & Region II & Region III & \multicolumn{2}{|r|}{ Region IV } \\
\hline \multicolumn{2}{|c|}{ Type A } & \multicolumn{2}{|c|}{$\begin{array}{l}\text { Forward slip to } \\
\text { crack tip. }\end{array}$} & \multicolumn{2}{|c|}{$\begin{array}{l}\text { Crack tip sticks. } \\
\text { Backslip zone } \\
\text { to tip. Crack } \\
\text { tip opens. } K_{n} \\
\text { becomes less. }\end{array}$} & $\begin{array}{l}\text { Forward slip to } \\
\text { tip. Crack } \\
\text { closes. }\end{array}$ & $\begin{array}{l}\text { Surface stick } \\
\text { zone. } \\
\text { Continued } \\
\text { forward slip } \\
\text { to tip for rest } \\
\text { of crack. }\end{array}$ & \multicolumn{2}{|c|}{$\begin{array}{l}\text { Stick. Small zone } \\
\text { of backslip at } \\
\text { surface if }|\lambda| \\
>2 f .\end{array}$} \\
\hline \multicolumn{2}{|c|}{ Type $B$} & \multicolumn{2}{|c|}{$\begin{array}{l}\text { Forward slip to } \\
\text { crack tip. }\end{array}$} & \multicolumn{2}{|c|}{$\begin{array}{l}\text { Forward slip to } \\
\text { tip. Crack tip } \\
\text { opens. }\end{array}$} & $\begin{array}{l}\text { Gap/forward/ } \\
\text { stick zone } \\
\text { recedes to } \\
\text { surface. } \\
\text { Crack closes. }\end{array}$ & $\begin{array}{l}\text { Crack may stick } \\
\text { along length } \\
\text { or forward } \\
\text { slip from tip } \\
\text { up towards } \\
\text { surface. }\end{array}$ & \multicolumn{2}{|c|}{$\begin{array}{l}\text { Continued stick. } \\
\text { Full stick. }\end{array}$} \\
\hline & $c / L$ & $f$ & $K_{\mathrm{u}} / p_{0} \sqrt{\pi c}$ & $K_{n} / p_{0} \sqrt{\pi c}$ & $\lambda$ & $K_{n} / p_{0} \sqrt{\pi c}$ & $K_{11} / p_{0} \sqrt{\pi c}$ & $\lambda$ & $K_{\mathrm{II}} / p_{0} \sqrt{\pi c}$ \\
\hline $\begin{array}{l}A \\
A \\
B \\
B \\
B\end{array}$ & $\begin{array}{l}0.3 \\
1.0 \\
1.0 \\
1.0 \\
0.5\end{array}$ & $\begin{array}{l}0.4 \\
0.4 \\
0.79 \\
0.7 \\
0.7\end{array}$ & $\begin{array}{l}0.168 \\
0.163 \\
0.067 \\
0.091 \\
0.067\end{array}$ & $\begin{array}{l}\text { sticks } \\
\text { sticks } \\
0.112 \\
0.112 \\
0.158\end{array}$ & $\begin{array}{l}0.41 \\
0.60 \\
0.60 \\
0.60 \\
0.49\end{array}$ & $\begin{array}{l}0.169 \\
0.164 \\
\text { sticks } \\
\text { sticks } \\
\text { sticks }\end{array}$ & $\begin{array}{l}0.198 \\
0.210 \\
0.123 \\
0.143 \\
\text { sticks }\end{array}$ & $\begin{array}{l}-0.41 \\
-0.60 \\
-0.60 \\
-0.60 \\
-0.49\end{array}$ & $\begin{array}{l}\text { sticks } \\
\text { sticks } \\
\text { sticks } \\
\text { sticks } \\
\text { sticks }\end{array}$ \\
\hline
\end{tabular}

intensity $\left(K_{1}\right)$ is also experienced. The tip of a type $A$ crack initially sticks as an infinitesimal shear traction is applied. Further increase in the value of $\lambda$ gives rise to a backslip zone in the neighborhood of the crack tip, so that the mode Il stress intensity factor is reduced and may even become negative if $c / L$ is small. As for type $B$ cracks, when $a \rightarrow c$ an opening mode stress intensity is also experienced.

\section{REGION II}

If the crack tip is experiencing mode 1 loading, the initial part of the unloading (region II) will be reversible, regardless of whether the crack is of type $A$ or $B$. Thus, the loading and unloading paths will be identical until the crack faces come into contact and frictional effects introduce irreversibilities. The value of the mode Il stress intensity factor at the onset of closure is indicated by the dashed line in Fig. 2. The line separating types $A$ and $B$ also gives the value of $\lambda$ at this point, if the value of $f$ given on the ordinate is interpreted as $\lambda$.

For type $A$ cracks, further unloading leads to forward slip between the contacting crack faces, and results in a positive increase in the mode II stress intensity factor. Figure 3 shows the values when all surface shear tractions have been removed, i.e. $\lambda$ $=0$. As forward slip occurs along the entire crack face in region Il, the calculation is essentially the same as that describing forward slip, which is detailed in Part 1[1] and will not be repeated here.

The response of type $B$ cracks to a reduction in the value of $\lambda$ is more complicated. It was found that the crack faces stick over most of their length, while a small portion adjacent to the closure point remains in a state of forward slip. As the shear load $\lambda p_{0}$ is removed, any point on the crack faces within the initial gap experiences closure with forward slip and then subsequently stick as $\lambda$ is reduced to zero. This configuration must be solved incrementally because the dislocation density at a point in the stick zone can only be determined from that previous stage in the process when the point in question experienced the transition from slip to stick. Recently, Dundurs and Gautesen[3] solved a problem of this type involving two half-spaces pressed together and locally separated by two point forces travelling at constant speed. In their analysis it was possible to write down in closed form an expression for the normal traction across the crack face, and this facilitated a solution for $\dot{B}_{x}(\zeta)$, the derivative of dislocation density with respect to time. This is not possible for the present problem, since the 


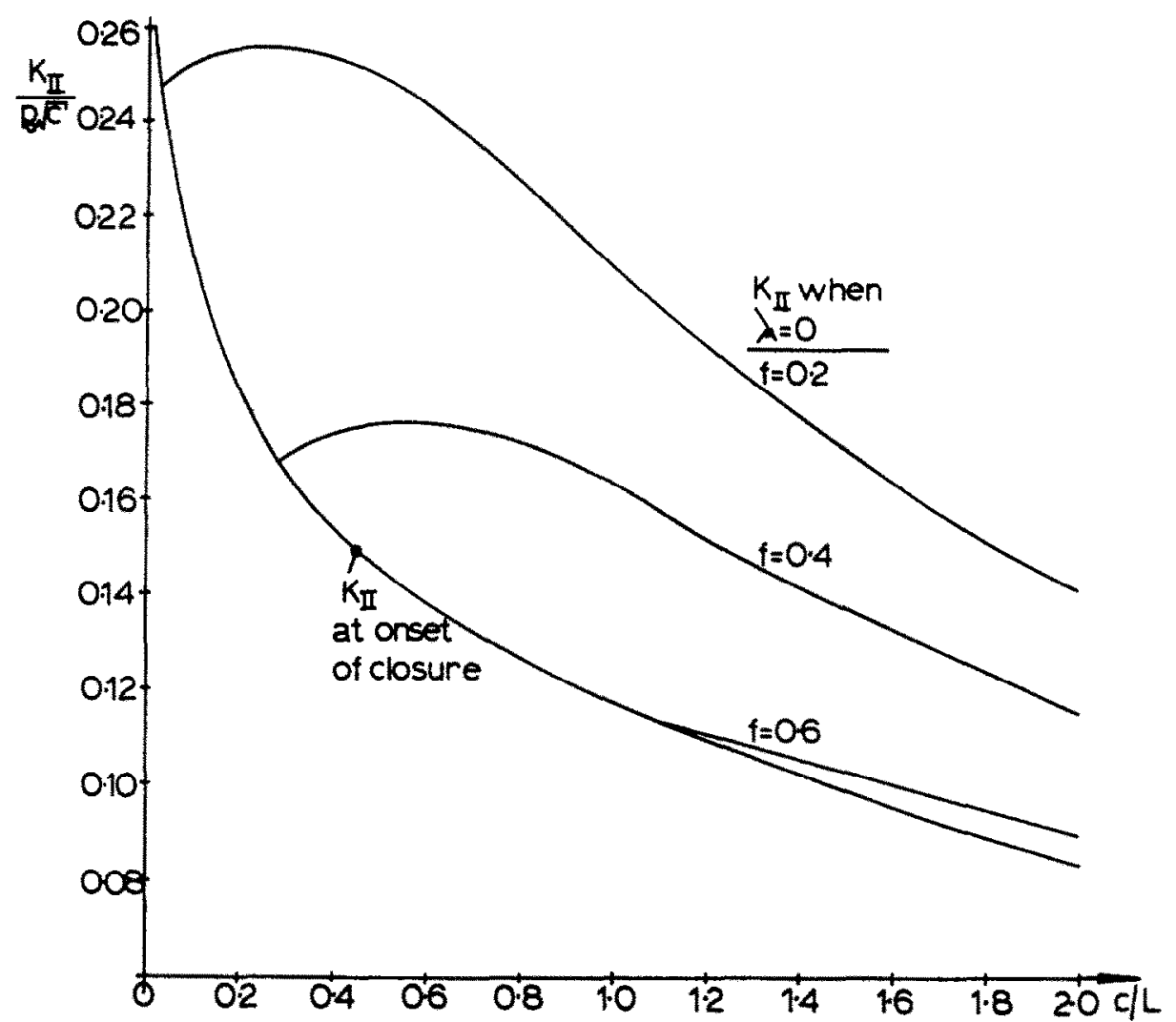

Fig. 3. Stress intensity factor for type $A$ cracks at the end of region 11 . Points to the left below the bounding curve correspond to type $B$ solutions.

normal traction $N(x)$ must itself be written as an integral equation in terms of distributed climb dislocations[1], and an alternative procedure is adopted which involves unloading in a series of increments.

Suppose that the unloading process has been solved to some general $(i-1)$ th stage, at which the end of the separation zone and the stick-slip transition point are denoted by $a_{i-1}, b_{i-1}$, respectively, as shown in Fig. 4(a). At this point the shear and normal interfacial tractions $S(x)$ and $N(x)$ are given by

$$
\begin{aligned}
N_{i-1}(x) & =\left(g_{2}(x)+\lambda_{i-1} g_{3}(x)\right) p_{0}+\int_{0}^{a_{i-1}} B_{y}(\zeta) K(x, \zeta) \mathrm{d} \zeta \\
S_{i-1}(x) & =-f N_{i-1}, \quad 0 \leq x \leq b_{i-1}
\end{aligned}
$$
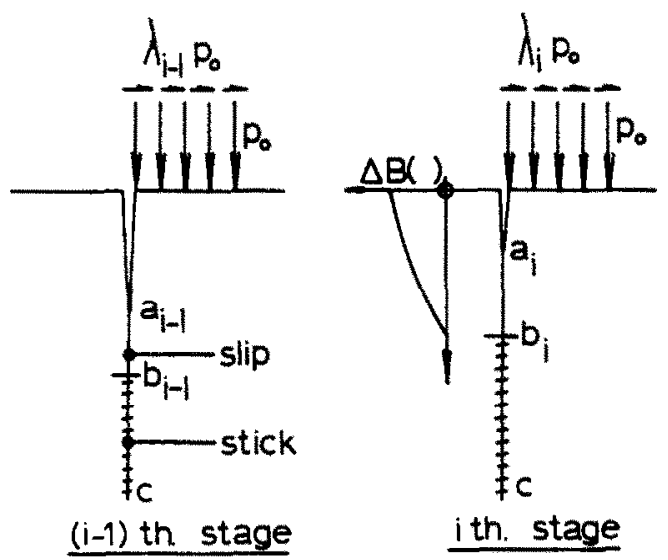

Fig. 4. Incremental formulation for type $B$ cracks, region II. 
where the functions $g_{2}(x), g_{3}(x)$ are the bilateral (Flamant) solutions die to normal and tangential tractions, respectively, and the kernel $K(x, \zeta)$ gives the normal stress at point $x$ due to a unit climb dislocation at point $\zeta$.

The distribution of climb dislocations, $B_{y}(\zeta)$ is found by imposing the condition

$$
N_{i-1}(x)=0, \quad 0 \leq x \leq a_{i-1},
$$

and choosing the bounded solution to the resulting singular integral equation, since there is a smooth transition between the gap and the closed portion of the crack, as discussed in [5]. To solve for the ith stage [Fig. 4(b)], we first need to establish the new normal traction distribution. This is possible as the normal and shear tractions are uncoupled for this problem, i.e. climb dislocations do not give rise to a shear stress on the crack, and glide dislocations do not give rise to normal stress. We then add an array of glide dislocations $\Delta B_{x}(\zeta)$ in the interval $0<x<b_{i}$ to restore the slip condition; i.e.

$$
\begin{aligned}
S_{i}(x) & =S_{i-1}(x)+p_{0} g_{2}\left(\lambda_{i}-\lambda_{i-1}\right)+\int_{0}^{b_{i}} \Delta B_{x}(\zeta) K(x, \zeta) \mathrm{d} \zeta \\
& =-f N_{i}, \quad 0 \leq x \leq b_{i},
\end{aligned}
$$

where $K(x, \zeta)$ now gives the shear traction at point $x$ due to a unit glide dislocation at point $\zeta$. Thus, we seek a solution to the integral equation

$$
\int_{0}^{b_{i}} \Delta B_{x}(\zeta) K(x, \zeta) \mathrm{d} \zeta=-p_{0} g_{2}(x)\left(\lambda_{i}-\lambda_{i-1}\right)-f\left(N_{i}(x)-N_{i-1}(x)\right), \quad 0 \leq x \leq b_{i}
$$

Since slip is giving way to stick, a bounded solution is required[4], and this yields an extra equation, enabling $b_{i}$ to be found. Note that the singular integral equation (1) for normal tractions is Cauchy only in the interval $0<x<a_{i-1}$, so that we are free to choose collocation points appropriate to the bounded integral (5) in the range $a_{i-1}<$ $x<b_{i}$, and $N_{i-1}(x)=0$, for $0<x<a_{i-1}$. A running total is kept of the shear stresses present in the stick zone as $\lambda$ is reduced to zero. The residual shear tractions present when $\lambda$ has once again become zero are shown in Fig. 5 for representative cases.

An obvious disadvantage with this technique is that the continuous variation of $B_{x}(\zeta), B_{y}(\zeta)$ and $b$ with $\lambda$ cannot be represented, though the error was reduced by averaging the $\Delta B_{x}(\zeta)$ contribution from adjacent steps, equivalent to assuming a piecewise-linear variation of these variables. The problem is aggravated by the presence of "hooks" in the shear stress distribution[4].

It was found that for long cracks $(c / L>1.0)$ and coefficients of friction just above the critical line of Fig. 2, there was a tendency for a second region of forward slip to develop near the crack tip as $\lambda$ approached zero. A formal analysis of these cases would involve simultaneously adding glide dislocations in two zones and coupled iterations between these two zones to find the extent of the stick zone; therefore, they were not pursued. The necessity of using an incremental solution for type $B$ cracks means that it is not feasible to obtain a comprehensive range of results, owing to limitations on computing time.

\section{REGION III}

For type $A$ cracks the starting conditions at the onset of reversed loading are

$$
\begin{aligned}
N(x) & =p_{0} g_{2}(x), \\
S(x) & =-f N(x)=-p_{0} f g_{2}(x) .
\end{aligned}
$$




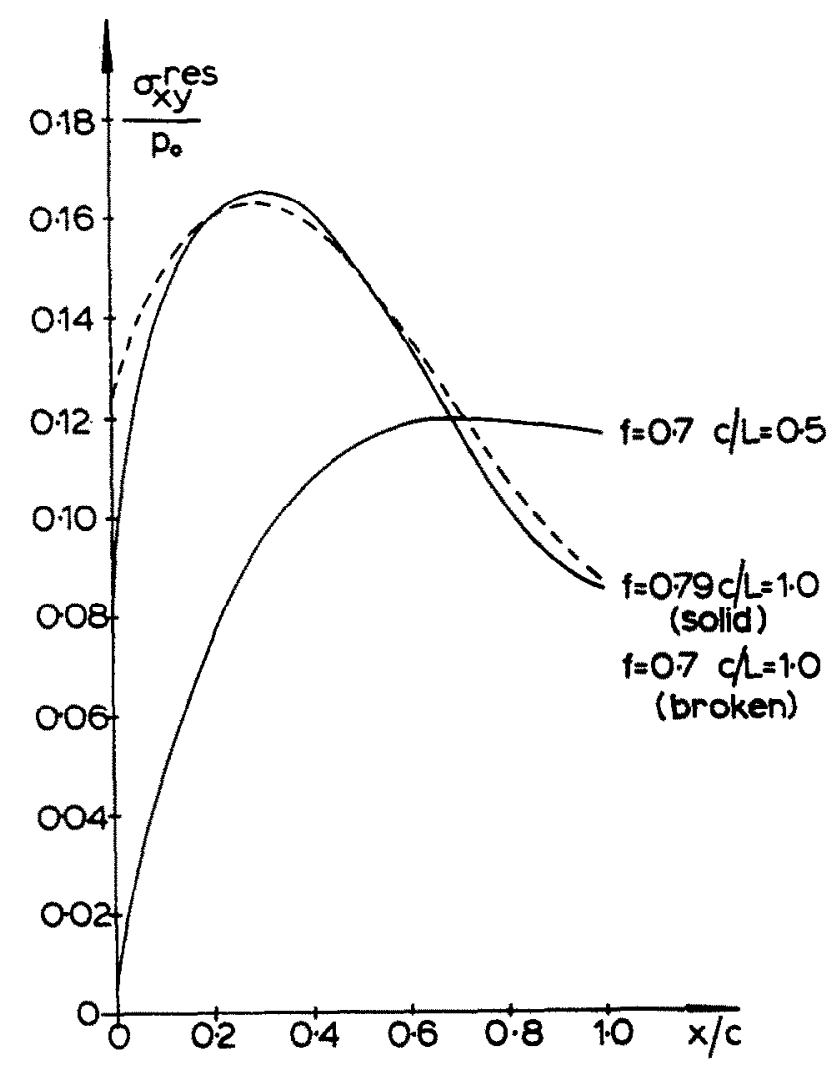

Fig. 5. Curves showing typical residual curve tractions at the end of region 11 for type $B$ cracks.

Subsequently, the normal stress is given by

$$
N(x)=p_{0}\left(g_{2}(x)+\lambda g_{3}(x)\right) .
$$

It should be noted that $g_{3}(x)$ has a logarithmic singularity at $x=0$, so that for any $\lambda$ $<0$ there will be a zone of very high compression near the surface and, hence, a stick region, as depicted in Fig. 6(a). It is instructive to formulate a solution on the basis of
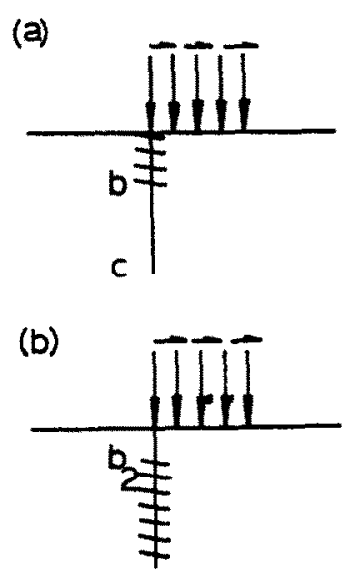

Fig. 6. Crack geometries to be solved: (a) for type $A$ cracks, region III; (b) for type $B$ cracks, region IV. 
the problem being incremental. At the $(i-1)$ th stage we have

$$
\begin{aligned}
N_{i-1}(x) & =p_{0}\left(g_{2}(x)+\lambda_{i-1} g_{3}(x)\right), \\
S_{i-1}(x) & =-f p_{0}\left(g_{2}(x)+\lambda_{i-1} g_{3}(x)\right) ; \quad b_{i-1} \leq x \leq c .
\end{aligned}
$$

At the $i$ th stage,

$$
\begin{aligned}
S_{i}(x) & =S_{i-1}(x)+\left(\lambda_{i}-\lambda_{i-1}\right) p_{0} g_{2}(x)+\int_{b_{i}}^{c} \Delta B_{x}(\zeta) K(x, \zeta) \mathrm{d} \zeta \\
& =-f p_{0}\left(g_{2}(x)+\lambda_{i} g_{3}(x)\right), \\
\int_{b_{i}}^{c} \Delta B_{x}(\zeta) K(x, \zeta) \mathrm{d} \zeta & =-p_{0}\left(g_{2}(x)+f g_{3}(x)\right)\left(\lambda_{i}-\lambda_{i-1}\right), \quad b_{i}<x<c .
\end{aligned}
$$

The right-hand side of eqn (12) is simply proportional to the increment in $\lambda$; hence the solution for $\Delta B_{x}(\zeta)$, which is singular at $c$ and bounded at $b_{i}$, is also proportional to the increment, and the value of $b$ is constant (i.e. $b_{i}=b_{i-1}$ ). Similarly, the value of $K_{11}$ is also proportional to the change in $\lambda$. Results for the change in $K_{11}$ resulting from full reloading through region III and for the extent of the stick zone are given in Fig. 7 as functions of the interfacial friction coefficient and crack length. Values for coefficients of friction of 0.5 and 0.6 are shown only for large cracks because shorter ones are relevant to type $B$ (see Figs. 2 and 3). Low coefficients of friction values are not shown for long cracks since $b$ becomes extremely small, and there are difficulties in attaining sufficient numerical accuracy.

Type $B$ cracks may experience stick throughout region III, or may forward slip, depending on the coefficient of interfacial friction and crack length. Detailed numerical calculations are necessary to establish the slip condition, but we may derive a sufficient condition for there to be no slip. At the end of region Il the interfacial tractions satisfy

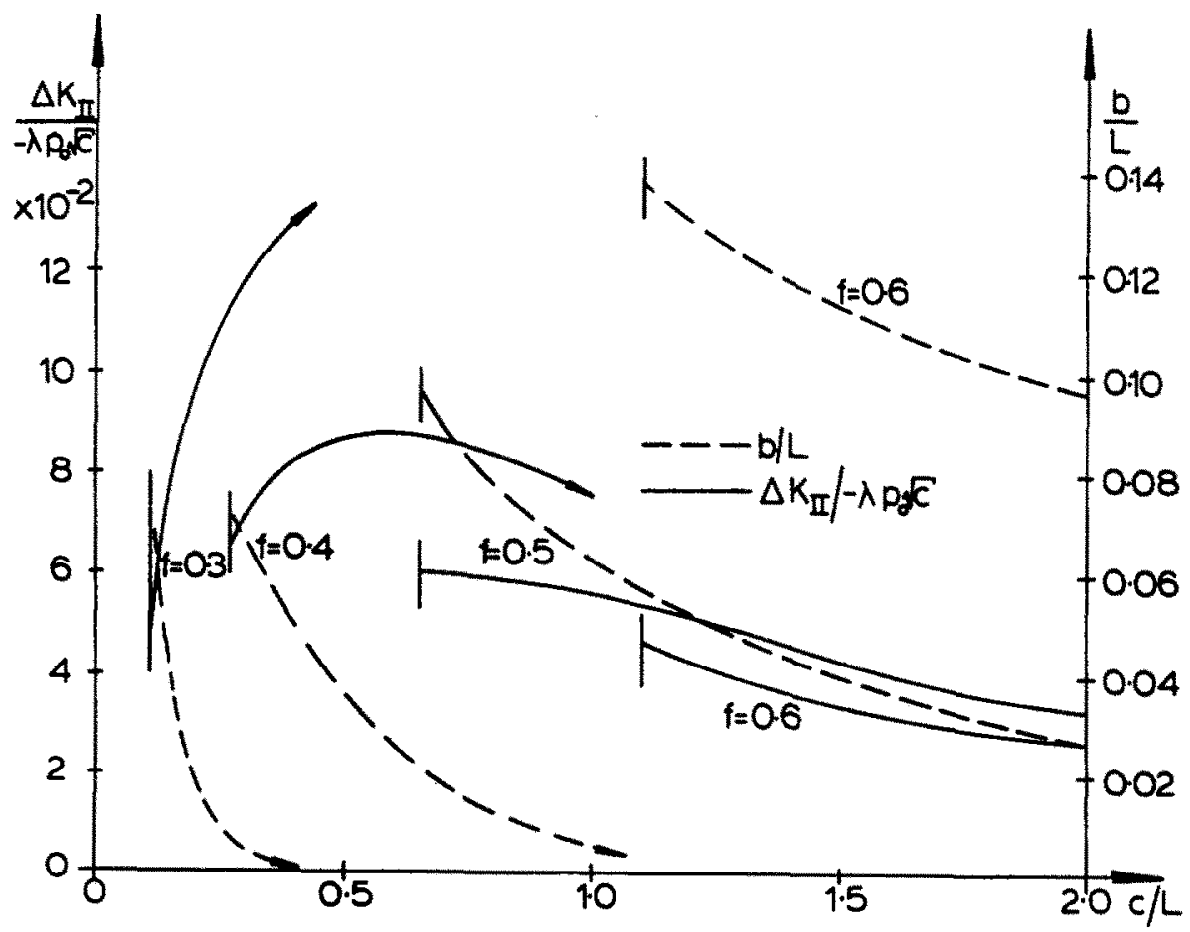

Fig. 7. Response of type A cracks, region III. Shown is the change in stress intensity factor with $c / L$, and the size of the surface stick zone. 
the inequalities

$$
0<S(x)<-f N(x), \quad 0 \leq x \leq c .
$$

Hence, if the increments in shear and normal tractions satisfy the inequalities

$$
0<\Delta S(x)<-f \Delta N(x)
$$

there will certainly be no further forward slip. This is ensured if

$$
f>-g_{2}(c) / g_{3}(c),
$$

since the ratio $g_{2}(x) / g_{3}(x)$ increases monotonically with $x$. The region where inequality (15) is satisfied is denoted by $B_{1}$ in Fig. 2.

At the end of region Il, it was found that in every case which was treated the crack tip showed the greatest tendency to slip forwards. It would seem, then, that if there is to be forward slip for type $B$ cracks in region III, it will start at the crack tip and gradually extend towards the surface. This configuration is shown in Fig. 6(a). Thus, we have

$$
S(x)=S^{*}(x)+\lambda p_{0} g_{2}(x)+\int_{b}^{c} \Delta B_{x}(\zeta) K(x, \zeta) \mathrm{d} \zeta=-f N(x), \quad b \leq x \leq c,
$$

where $S^{*}(x)$ denotes the initial shear traction distribution resulting from region II analysis, the normal traction $N(x)=\left(p_{0} g_{2}(x)+\lambda g_{3}(x)\right)<0$ and, hence, the crack remains

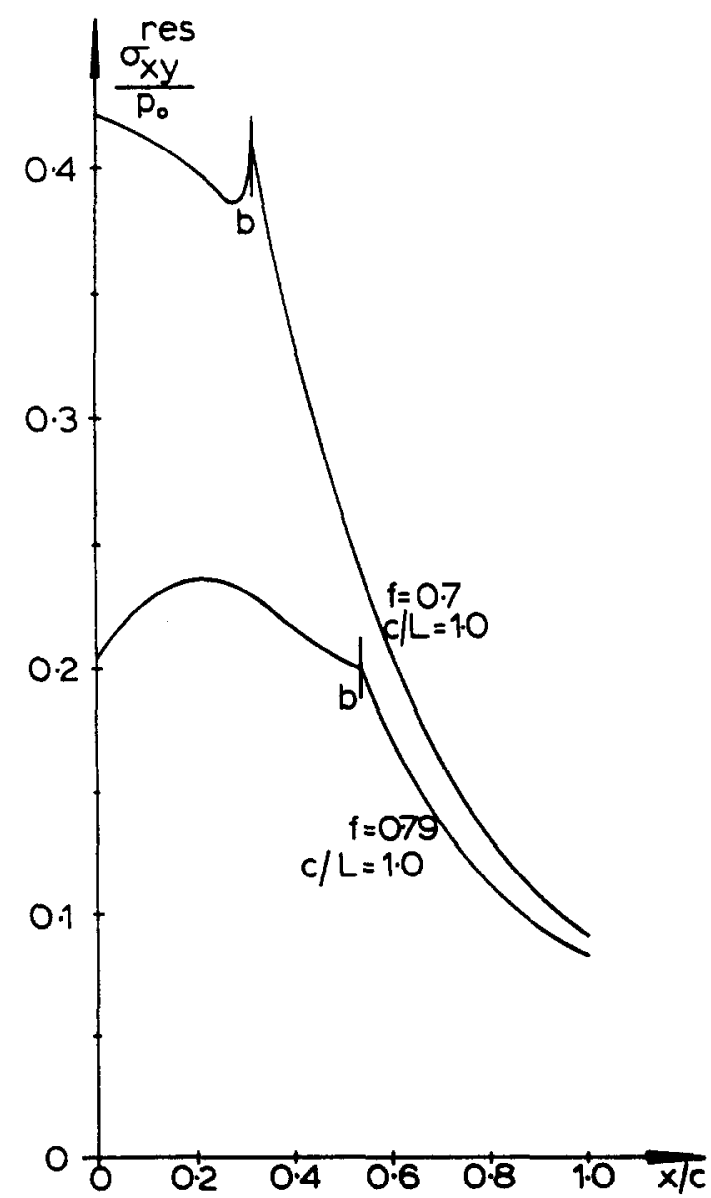

Fig. 8. Shear stresses remaining at the end of region III for type $B$ cracks. 
closed throughout regions 111 and IV. We seek a distribution for $\Delta B_{x}(\zeta)$ which is singular at $c$ but bounded at $b$. It was found that for a crack length of $c / L=0.5$ and $f=0.7$, the crack continued to stick everywhere, until $\lambda$ was less than -0.6 , even though inequality (15) does not hold. For the two cracks of length $c / L=1.0$ considered, forward slip did occur, and the shear traction distributions are given for $\lambda=-0.6$ at the end of region III in Fig. 8.

\section{REGION IV}

The simpler cracks to consider are those of type $A$. It will be recalled that at the end of region III they are in a state of forward slip along most of their length [Figs. 6(a) and 7], so that such a crack will stick or backslip as the value of $\lambda$ is increased to zero. It may be easily verified that further forward slip is impossible: suppose that some applied surface shear traction has been removed, so that $\lambda_{\max }<\lambda<0$. We continue to assume forward slip and recall that the solution is still proportional to $\Delta \lambda$. Thus, the same state of tractions exists as in region III, for any given $\lambda$, but the slip direction is now inconsistent with the change in $\lambda$; hence forward slip is impossible.

Backslip will occur unless, when $\lambda$ is reduced to zero,

$$
S(x)>f N(x)
$$

i.e.

$$
-p_{0} f\left(g_{2}(x)+\lambda_{\max } g_{3}(x)\right)-p_{0} \lambda_{\max } g_{2}(x)>f p_{0} g_{2}(x) .
$$

This must hold for all $\lambda$ in the range $\lambda_{\max }<\lambda<0$ and all $x$ in the range $b<x<c$. A violation will first occur at $b$. This places the requirement that, for no backslip,

$$
\left|\lambda_{\max }\right|<2 f
$$

It will be recognized that in practice $\lambda_{\max }$ is likely to be limited by the coefficient of friction between the contacting bodies. Since this will probably be of the same order as $f$, this inequality should hold, although no account has been taken of what might happen within the tiny stick zone present in region III [Fig. 6(a)].

A limited investigation was made of the extent of the backslip zone when inequality (18) is violated. Suppose that the stresses along the crack face at the end of region III are given by

$$
S^{*}(x)=-p_{0} f\left(g_{2}(x)+\lambda_{\max } g_{3}(x)\right)
$$

From the form of the bilateral solutions it would appear that any backslip would start at $b$ and propagate both upwards and downwards slightly, reaching the surface as $\lambda \rightarrow$ 0 (and hence $N(0)$ becomes finite) [Fig. 6(b)]. Thus, when all surface shear tractions have been removed, we may write

$$
\begin{aligned}
S(x) & =S^{*}(x)-\lambda_{\max } p_{0 g_{2}}(x)+\int_{0}^{b_{2}} B_{x}(\zeta) K(x, \zeta) \mathrm{d} \zeta=f N(x), \quad 0<x<b_{2}, \\
N(x) & =p_{0 g_{2}}(x) .
\end{aligned}
$$

The solution for $B_{x}(\zeta)$ is bounded at $b_{2}$. For crack lengths between $c / L=0.4$ and 1.0 , $\left|\lambda_{\max }\right|=1.0, f=0.4$, it was found that $b_{2} / L$ was about 0.20 . If the coefficient of friction was taken to be $0.3, b_{2} / L$ fell to 0.16 . Very short cracks, where a change in sign of the shear stress intensity factor would occur, were not considered.

Type $B$ cracks of length $c / L=1.0$ and coefficient of friction $f=0.7$ or 0.79 were considered, and these continued to stick. 


\section{CONCLUSION}

Within the limitations imposed by the incremental solution of type $B$ cracks, it is possible to follow the stress intensity factor for a wide range of cracks during a load cycle. It should be emphasized that the treatment presented relates to cracks which are fully open during part of the loading cycle, and thus a general cycle in the loading history is the same as the first, as all residual shear tractions vanish during the time the crack faces are apart. As an example, the stress intensity factors experienced during one cycle by five sample cracks are appended to Table 1.

Acknowledgement-David Hills gratefully acknowledges support received from the English Speaking Union during this work.

\section{REFERENCES}

1. D. A. Hills and Maria Comninou, An analysis of fretting fatigue cracks. I. Loading phase. Int. J. Solids Struct. under review.

2. A. J. McEvily, The microstructure and design of alloys. In Proc. 3rd Int. Conf. on the Strength of Metals and Alloys, Vol. 2. Cambridge (1973).

3. J. Dundurs and A. K. Gautesen, On the approach to steady state for frictional contact under moving loads. J. Appl. Mech. 50, 783-788 (1983).

4. Maria Comninou, J. R. Barber and J. Dundurs, Interface slip caused by a surface load moving at constant speed. Int. J. Mech. Sci. 25, 41-46 (1983).

5. J. Dundurs and Maria Comninou, Some consequences of the inequality conditions in contact and crack problems. J. Elasticity 9, 71-82 (1979). 